MATHEMATICS OF COMPUTATION

Volume 71, Number 238, Pages 527-535

S $0025-5718(01) 01334-5$

Article electronically published on May 22, 2001

\title{
ON THE STABILITY OF A FAMILY OF FINITE ELEMENT METHODS FOR HYPERBOLIC PROBLEMS
}

\author{
GERARD R. RICHTER
}

\begin{abstract}
We consider a family of tensor product finite element methods for hyperbolic equations in $R^{N}, N \geq 2$, which are explicit and generate a continuous approximate solution. The base case $N=2$ (an extension of the box scheme to higher order) is due to Winther, who proved stability and optimal order convergence. By means of a simple counterexample, we show that, for linear approximation with $N \geq 3$, the corresponding methods are unstable.
\end{abstract}

\section{INTRODUCTION}

We begin by describing, in the context of a simple model problem, a finite element method for hyperbolic equations in $R^{2}$ due to Winther [11. Our problem will be:

$$
\begin{aligned}
& u_{\alpha}=f(\mathbf{x}), \quad \mathbf{x}=\left(x_{1}, x_{2}\right) \in \Omega, \\
& u \text { given on } \Gamma_{\text {in }}(\Omega),
\end{aligned}
$$

where $\Omega$ is the unit square and $u_{\alpha} \equiv \alpha \cdot \nabla u$, where $\alpha=\left(\alpha_{1}, \alpha_{2}\right)$ is a unit vector consisting of positive constants. We denote the boundary of $\Omega$ by $\Gamma(\Omega)$, the unit outer normal by $\mathbf{n}=\left(n_{1}, n_{2}\right)$, and the inflow portion of $\Gamma(\Omega)$, where $\alpha \cdot \mathbf{n}<0$, by $\Gamma_{\text {in }}(\Omega)$. For our problem, $\Gamma_{\text {in }}(\Omega)$ consists of the $x_{1}=0$ and $x_{2}=0$ sides of $\Omega$. Likewise, $\Gamma_{\text {out }}(\Omega)$ is characterized by $\alpha \cdot \mathbf{n}>0$.

To apply Winther's scheme to (1.1), we first partition $\Omega$ into a mesh of rectangles $\mathcal{E}_{i_{1}, i_{2}}, 0 \leq i_{1} \leq n_{1}, 0 \leq i_{2} \leq n_{2}$, as illustrated in Figure 1 . Over this mesh, the solution to (1.1) can be developed one element at a time, first in $\mathcal{E}_{0,0}$, then in either $\mathcal{E}_{1,0}$ or $\mathcal{E}_{0,1}$, etc. For computational and theoretical purposes, it is useful to think of $u_{h}$ as evolving though the mesh in "layers", first in $S_{0}=\mathcal{E}_{0,0}$, then in $S_{1}=\mathcal{E}_{1,0} \cup \mathcal{E}_{0,1}$, then in $S_{2}=\mathcal{E}_{2,0} \cup \mathcal{E}_{1,1} \cup \mathcal{E}_{0,2}$, etc. Note that $u_{h}$ may be computed concurrently in the elements comprising a layer. The $j^{\text {th }}$ such layer is given by $S_{j}=\left\{\mathcal{E}_{i_{1}, i_{2}} \mid i_{1}+i_{2}=j\right\}$. After $u_{h}$ has been developed in $\Omega_{i} \equiv \bigcup_{j \leq i} S_{j}$, it will have advanced to the "frontline" $\mathcal{F}_{i}$, where

$$
\mathcal{F}_{i}=\left(\Gamma_{\text {in }}(\Omega)-\Gamma_{\text {in }}\left(\Omega_{i}\right)\right) \cup \Gamma_{\text {out }}\left(\Omega_{i}\right), \quad i=0,1, \ldots, n_{1}+n_{2} .
$$

The finite element approximation $u_{h}$ is developed in the same manner. For a generic rectangular element $\mathcal{E}=\mathcal{E}_{i_{1}, i_{2}}$, we denote by $\Pi_{k_{1}, k_{2}}(\mathcal{E})$ the corresponding tensor product space of polynomials of degree $k_{1}, k_{2}$ in $x_{1}, x_{2}$. The approximation subspace $S_{h}$ will consist of those $C^{0}(\Omega)$ functions which reduce to members of

Received by the editor December 8, 1999 and, in revised form, August 8, 2000.

2000 Mathematics Subject Classification. Primary 65M60, 65M12.

Key words and phrases. Finite elements, hyperbolic, explicit.

(C)2001 American Mathematical Society 


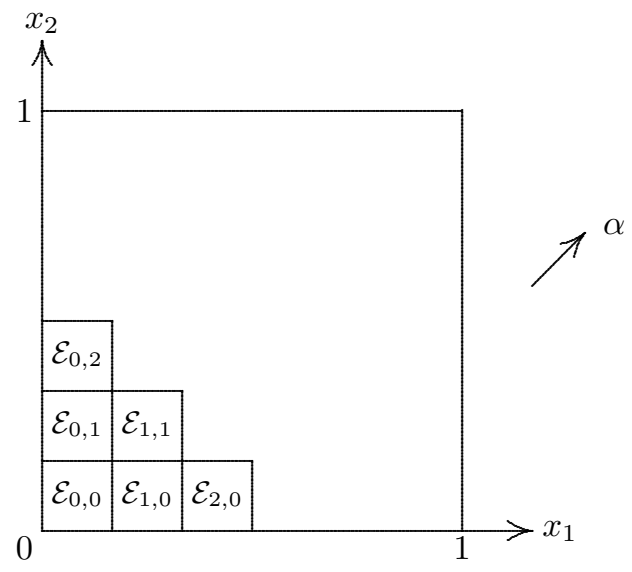

Figure 1.

$\Pi_{k, k}(\mathcal{E})$ in all elements $\mathcal{E}$. The finite element approximation $u_{h} \in S_{h}$ starts as an interpolant of the given initial data on $\Gamma_{\mathrm{in}}(\Omega)$, and is required to satisfy the conditions

$$
\left(\left(u_{h}\right)_{\alpha}, v_{h}\right)_{\mathcal{E}}=\left(f, v_{h}\right)_{\mathcal{E}}, \quad \text { for all } v_{h} \in \Pi_{k-1, k-1}(\mathcal{E}),
$$

where $(,)_{\mathcal{E}}$ denotes the $L^{2}(\mathcal{E})$ inner product. The number of equations equals the number of unknowns in $(1.3)$ because at the time when $u_{h}$ is computed in $\mathcal{E}$, it will already be known on $\Gamma_{\text {in }}(\mathcal{E})$.

This finite element method has an obvious extension to the more general problem where $\Omega$ is a hypercube in $R^{N}$ and $\alpha=\left(\alpha_{1}, \ldots, \alpha_{N}\right)>0$. For a generic element $\mathcal{E}=\mathcal{E}_{i_{1}, i_{2}, \ldots, i_{N}}$, the trial and test spaces are $N$-dimensional tensor product spaces $\Pi_{k, \ldots, k}(\mathcal{E})$ and $\Pi_{k-1, \ldots, k-1}(\mathcal{E})$ in $x_{1}, \ldots, x_{N} ;(1.3)$ thus becomes

$$
\left(\left(u_{h}\right)_{\alpha}, v_{h}\right)_{\mathcal{E}}=\left(f, v_{h}\right)_{\mathcal{E}}, \quad \text { for all } v_{h} \in \Pi_{k-1, \ldots, k-1}(\mathcal{E}) .
$$

The $j^{\text {th }}$ layer is now $S_{j}=\left\{\mathcal{E}_{i_{1}, i_{2}, \ldots, i_{N}} \mid i_{1}+\cdots+i_{N}=j\right\}$.

Explicit finite element methods such as these are potentially attractive candidates for hyperbolic systems arising in applications. As compared to the "canonical" explicit method, discontinuous Galerkin 6, 9, they use fewer unknowns. For example, if the $N=3$ version of (1.4) is applied with $k=1$ (linear approximation), there is only one unknown to be solved for per element vs. eight for the discontinuous Galerkin method. The $k=1$ version of the scheme (1.4) is equivalent to the box scheme [10] and serves as a basic ingredient in cell vertex methods, a class of finite volume methods for hyperbolic and convection-diffusion problems, and more general flow problems. The sole test function for linears, $v_{h}=1$, enforces conservation over elements $\mathcal{E}$. Error estimates have been obtained for cell vertex schemes in two dimensions [1, 8]. Several second-order-accurate schemes in three dimensions, including the $N=3, k=1$ version of (1.4), have been proposed in [2]. In both two and three dimensions, these schemes are known to admit spurious oscillatory solutions which neither grow nor decay. The method (1.4) also has potential applicability to transient hyperbolic systems. Here an easy way to obtain an explicit space-time mesh, suitable for application of (1.4), would be to start with hypercube elements $\mathcal{E}$, oriented so that two opposite vertices lie along a line parallel to the 
$t$-axis, and then compress or stretch in the $t$-direction [5]. This produces elements which are parallelograms for $N=2$, parallelepipeds for $N=3$, etc., to which the method (1.4) generalizes trivially.

For the base case $N=2$, the method (1.4) is known to be stable for arbitrary $k \geq 1$ and satisfies the bound

$$
\left|u_{h}\right|_{\Gamma_{\text {out }}(\Omega)}^{2}+\left\|u_{h}\right\|_{\Omega}^{2} \leq C\left(\left|u_{h}\right|_{\Gamma_{\text {in }}(\Omega)}^{2}+\|f\|_{\Omega}^{2}\right),
$$

where $|\cdot|$ and $\|\cdot\|$ denote boundary and interior $L^{2}$ norms, respectively, and $C$ denotes a generic constant, independent of mesh size $h$ and $u$. Winther [11] proved stability for the $N=2$ method in the setting of a more general hyperbolic system and derived various optimal order error estimates and superconvergence results. Whether the higher-dimensional analog of the method has the same desirable attributes seems not to have been answered. In this paper, we provide a negative indication. We show by means of a counterexample that the method (1.4) is algebraically unstable; thus the bound (1.5) does not hold, for the case of linear approximation for any $N>2$. For smooth solutions, however, convergence rates do not appear to suffer.

We note the existence of a related family of explicit finite element methods 9 ] which generates a continuous approximate solution over simplices in $R^{N}$ as opposed to hypercubes. Stability and near-optimal order convergence have been proved for the case $N=2$ and an arbitrarily high degree of approximation 3, 4. Again, there is no analysis for $N>2$. In fact, it is not clear whether, for $N>2$, there exists any stable, optimal or near-optimal order finite element method for hyperbolic PDE's which is explicit and generates a continuous approximation.

In $\S 2$, we show that the bound (1.5) holds for $N=2$ and $k \geq 1$. This was first shown by Winther [1] for more general variable coefficient, linear hyperbolic systems. Ours is a simpler analysis aimed at achieving the stability bound (1.5) for the limited model problem (1.1). In $\S 3$, we reduce the $k=1$ version of (1.4) to a set of difference equations and construct a counterexample to (1.5) for $N>2$.

\section{Stability analysis For the CASE $N=2$}

We assume a quasi-uniform mesh of size $h$. A generic element $\mathcal{E}=\mathcal{E}_{i_{1}, i_{2}}$ for the case $N=2$ is shown in Figure 2. It will be convenient to parameterize $\Gamma_{\text {in }}(\mathcal{E})$ and $\Gamma_{\text {out }}(\mathcal{E})$ in terms of a cooordinate $t \equiv-\alpha_{2} x_{1}+\alpha_{1} x_{2}$ measured normal to the characteristic direction, as indicated in Figure 2. We use the notation $u_{h \text {, in }}=$ $\left.u_{h}\right|_{\Gamma_{\text {in }}(\mathcal{E})}, u_{h, \text { out }}=\left.u_{h}\right|_{\Gamma_{\text {out }}(\mathcal{E})}, u_{h, \text { in }}^{\prime}=\frac{d}{d t} u_{h, \text { in }}, u_{h, \text { out }}^{\prime}=\frac{d}{d t} u_{h, \text { out }}$. We also define $L^{2}$ projection operators

$$
\begin{aligned}
& Q_{1}: \Pi_{k, k}(\mathcal{E}) \rightarrow \Pi_{k-1, k}(\mathcal{E}), \\
& Q_{2}: \Pi_{k, k}(\mathcal{E}) \rightarrow \Pi_{k, k-1}(\mathcal{E}),
\end{aligned}
$$

and a boundary projection $Q$ which, for $\mathcal{D}=\Gamma_{\text {in }}(\mathcal{E})$ or $\mathcal{D}=\Gamma_{\text {out }}(\mathcal{E})$, maps $L^{2}(\mathcal{D})$ onto the space of discontinuous (in general) piecewise polynomials of degree $\leq k-1$ on the line segments comprising $\mathcal{D}$. In this section, all boundary norms will be taken with respect to $t$, and the notation $|\cdot|$ will signify an $L^{2}\left[t_{0}, t_{1}\right]$ norm of the indicated quantity. 


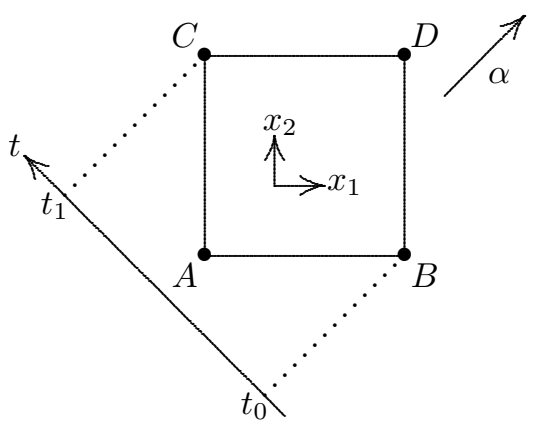

Figure 2.

Lemma 2.1. On $\mathcal{E}, u_{h}$ satisfies

$$
\begin{aligned}
& \left|u_{h, \text { out }}^{\prime}\right|^{2} \leq\left|u_{h, \text { in }}^{\prime}\right|^{2}+h^{-2}\left(\left\|u_{h}\right\|_{\mathcal{E}}^{2}+C\|f\|_{\mathcal{E}}^{2}\right) \\
& \left|Q u_{h, \text { out }}\right|^{2} \leq\left|Q u_{h, \text { in }}\right|^{2}+\left\|u_{h}\right\|_{\mathcal{E}}^{2}+\|f\|_{\mathcal{E}}^{2}, \\
& \left\|u_{h}\right\|_{\mathcal{E}}^{2} \leq C\left(h\left|u_{h, \text { in }}\right|^{2}+h^{2}\|f\|_{\mathcal{E}}^{2}\right) \\
& \left|Q u_{h, \text { out }}\right|^{2}+h^{2}\left|u_{h, \text { out }}^{\prime}\right|^{2}+\left\|u_{h}\right\|_{\mathcal{E}}^{2} \\
& \quad \leq(1+C h)\left(\left|Q u_{h, \text { in }}\right|^{2}+h^{2}\left|u_{h, \text { in }}^{\prime}\right|^{2}\right)+C\|f\|_{\mathcal{E}}^{2} .
\end{aligned}
$$

Proof. Letting $s$ denote arclength along $\Gamma(\mathcal{E})$, measured counterclockwise, we have $d t=\alpha \cdot \mathbf{n} d s$. Thus the choice $v_{h}=v_{h}^{*} \equiv 2 \alpha_{1} \alpha_{2}\left(u_{h}\right)_{x_{1} x_{2}}$ in (1.3) gives

$$
\begin{aligned}
\left(\left(u_{h}\right)_{\alpha}, v_{h}^{*}\right)_{\mathcal{E}} & =2 \alpha_{2}\left(\left(\alpha_{1} u_{h}\right)_{x_{1}},\left(\alpha_{1} u_{h}\right)_{x_{1} x_{2}}\right)_{\mathcal{E}}+2 \alpha_{1}\left(\left(\alpha_{2} u_{h}\right)_{x_{2}},\left(\alpha_{2} u_{h}\right)_{x_{1} x_{2}}\right)_{\mathcal{E}} \\
& =\int_{\overline{D C} \cup \overline{A B}}\left\{\left(\alpha_{1} u_{h}\right)_{x_{1}}\right\}^{2} \alpha_{2} n_{2} d s+\int_{\overline{B D} \cup \overline{C A}}\left\{\left(\alpha_{2} u_{h}\right)_{x_{2}}\right\}^{2} \alpha_{1} n_{1} d s \\
& =\alpha_{1}^{2} \alpha_{2}^{2} \int_{\overline{B D} \cup \overline{D C}}\left(u_{h, \text { out }}^{\prime}\right)^{2} d t-\alpha_{1}^{2} \alpha_{2}^{2} \int_{\overline{B A} \cup \overline{A C}}\left(u_{h, \text { in }}^{\prime}\right)^{2} d t \\
& =\alpha_{1}^{2} \alpha_{2}^{2}\left(\left|u_{h, \text { out }}^{\prime}\right|^{2}-\left|u_{h, \text { in }}^{\prime}\right|^{2}\right) .
\end{aligned}
$$

Via an inverse inequality, and the Schwarz and arithmetic-geometric mean $(|a b| \leq$ $\left.\frac{1}{2}\left(\theta a^{2}+\frac{b^{2}}{\theta}\right), \theta>0\right)$ inequalities, we obtain

$$
\left|\left(f, v_{h}^{*}\right)_{\mathcal{E}}\right| \leq C\|f\|_{\mathcal{E}} \cdot\left\|\left(u_{h}\right)_{x_{1} x_{2}}\right\|_{\mathcal{E}} \leq C h^{-2}\|f\|_{\mathcal{E}}\left\|u_{h}\right\|_{\mathcal{E}} \leq h^{-2}\left(\left\|u_{h}\right\|_{\mathcal{E}}^{2}+C\|f\|_{\mathcal{E}}^{2}\right),
$$
and (2.1) follows.

Taking $v_{h}=v_{h}^{* *} \equiv 2 Q_{1} Q_{2} u_{h}$ in (1.3), we get

$$
\begin{aligned}
\left(\left(u_{h}\right)_{\alpha}, v_{h}^{* *}\right)_{\mathcal{E}} & =2 \alpha_{1}\left(\left(u_{h}\right)_{x_{1}}, Q_{1} Q_{2} u_{h}\right)_{\mathcal{E}}+2 \alpha_{2}\left(\left(u_{h}\right)_{x_{2}}, Q_{2} Q_{1} u_{h}\right)_{\mathcal{E}} \\
& =2 \alpha_{1}\left(\left(Q_{2} u_{h}\right)_{x_{1}}, Q_{2} u_{h}\right)_{\mathcal{E}}+2 \alpha_{2}\left(\left(Q_{1} u_{h}\right)_{x_{2}}, Q_{1} u_{h}\right)_{\mathcal{E}} \\
& =\int_{\overline{B D} \cup \overline{C A}}\left(Q_{2} u_{h}\right)^{2} \alpha_{1} n_{1} d s+\int_{\overline{D C} \cup \overline{A B}}\left(Q_{1} u_{h}\right)^{2} \alpha_{2} n_{2} d s \\
& =\int_{\overline{B D} \cup \overline{D C}}\left(Q u_{h, \text { out }}\right)^{2} d t-\int_{\overline{B A} \cup \overline{A C}}\left(Q u_{h, \text { in }}\right)^{2} d t \\
& =\left|Q u_{h, \text { out }}\right|^{2}-\left|Q u_{h, \text { in }}\right|^{2} .
\end{aligned}
$$

Application of the Schwarz and arithmetic-geometric mean inequalities to $\left(f, v_{h}^{* *}\right)_{\mathcal{E}}$ then yields $(2.2)$. 
Uniqueness (hence existence) of $u_{h}$ within $\mathcal{E}$ may be established by showing that if $\left|u_{h, \text { in }}\right|=0$ and $\|f\|_{\mathcal{E}}=0$, then $\left\|u_{h}\right\|_{\mathcal{E}}=0$. This is trivial to show for the case of linear approximation $(k=1)$; more generally, see [1]. The bound (2.3) then follows by an appropriate scaling of norms.

To establish (2.4), we note the existence of constants $\gamma_{1}, \gamma_{2}$ such that

$$
\gamma_{1}\left|u_{h, \text { in }}\right|^{2} \leq\left|Q u_{h, \text { in }}\right|^{2}+h^{2}\left|u_{h, \text { in }}^{\prime}\right|^{2} \leq \gamma_{2}\left|u_{h, \text { in }}\right|^{2}
$$

with an analogous bound holding for $u_{h \text {,out }}$. Applying this to the sum of $(2.2), h^{2}$ times (2.1), and three times (2.3), we obtain (2.4).

Having proved the lemma, we now apply (2.4) over layer $S_{i}$ (cf. (1.2)):

$$
\left|Q u_{h}\right|_{\mathcal{F}_{i}}^{2}+h^{2}\left|u_{h}^{\prime}\right|_{\mathcal{F}_{i}}^{2}+\left\|u_{h}\right\|_{S_{i}}^{2} \leq(1+C h)\left(\left|Q u_{h}\right|_{\mathcal{F}_{i-1}}^{2}+h^{2}\left|u_{h}^{\prime}\right|_{\mathcal{F}_{i-1}}^{2}\right)+C\|f\|_{S_{i}}^{2} .
$$

Thus over the $O\left(h^{-1}\right)$ layers comprising $\Omega$ :

$$
\left|Q u_{h}\right|_{\Gamma_{\text {out }}(\Omega)}^{2}+h^{2}\left|u_{h}^{\prime}\right|_{\Gamma_{\text {out }}(\mathcal{E})}^{2}+\left\|u_{h}\right\|_{\Omega}^{2} \leq C\left(\left|Q u_{h}\right|_{\Gamma_{\text {in }}(\Omega)}^{2}+h^{2}\left|u_{h}^{\prime}\right|_{\Gamma_{\text {in }}(\Omega)}^{2}+\|f\|_{\Omega}^{2}\right) .
$$

The desired stability result (1.5) then follows from a suitable application of (2.5).

Before closing this section, we briefly consider the case $N=3$. Let $\mathcal{S}$ be the set of triplets $\{(1,2,3),(2,3,1),(3,1,2)\}$. The test functions $v_{h}^{*}$ and $v_{h}^{* *}$ used in the proof of Lemma 2.1 can be extended to $N=3$ as follows:

$$
\begin{aligned}
v_{h}^{*} & =2 \sum_{(i, j, k) \in \mathcal{S}} \alpha_{i} \alpha_{j} \mathcal{Q}_{k}\left(u_{h}\right)_{x_{i} x_{j}}, \\
v_{h}^{* *} & =2 \mathcal{Q}_{1} \mathcal{Q}_{2} \mathcal{Q}_{3} u_{h},
\end{aligned}
$$

where $\mathcal{Q}_{1}, \mathcal{Q}_{2}, \mathcal{Q}_{3}$ are the $N=3$ analogs of $Q_{1}, Q_{2}$ defined previously. It can be shown that these give the following boundary integrals:

$$
\begin{aligned}
& \left(\left(u_{h}\right)_{\alpha}, v_{h}^{*}\right)_{\mathcal{E}} \\
& \quad=\sum_{(i, j, k) \in \mathcal{S}} \int_{\Gamma(\mathcal{E})}\left[\left(Q_{j}\left(\alpha_{i} u_{h}\right)_{x_{i}}\right)^{2}+\left(\alpha_{i} u_{h}\right)_{x_{i}}\left(\alpha_{j} u_{h}\right)_{x_{j}}+\left(Q_{i}\left(\alpha_{j} u_{h}\right)_{x_{j}}\right)^{2}\right] \alpha_{k} n_{k} \\
& \left(\left(u_{h}\right)_{\alpha}, v_{h}^{* *}\right)_{\mathcal{E}}=\sum_{(i, j, k) \in \mathcal{S}} \int_{\Gamma(\mathcal{E})}\left(\mathcal{Q}_{i} \mathcal{Q}_{j} u_{h}\right)^{2} \alpha_{k} n_{k} .
\end{aligned}
$$

A key difference from the $N=2$ case is that the second derivatives appearing in $v_{h}^{*}$ now require projection into the test space. The result is that $u_{h}$ is not fully coerced on $\Gamma(\mathcal{E})$ by these two test functions.

\section{Demonstration of instability for $N>2$}

We now consider the $N$-dimensional method (1.4). We restrict our attention to a uniform mesh $\{\mathcal{E}\}$ of hypercubes of size $h$ in each coordinate direction and focus on the linear case, $k=1$, where, for any $N$, the only test function is $v_{h} \equiv 1$. To facilitate the description of the resulting method, we use a local coordinate system $\hat{\mathbf{x}}=\left(\hat{x}_{1}, \ldots, \hat{x}_{N}\right)$ such that the vertices $V$ of $\mathcal{E}$ correspond to $\hat{x}_{i}= \pm 1, i=1, \ldots, N$, and define $\hat{u}_{h}(\hat{\mathbf{x}})=u_{h}(\mathbf{x})$. Figure 3 contains an illustration for the case $N=3$. 


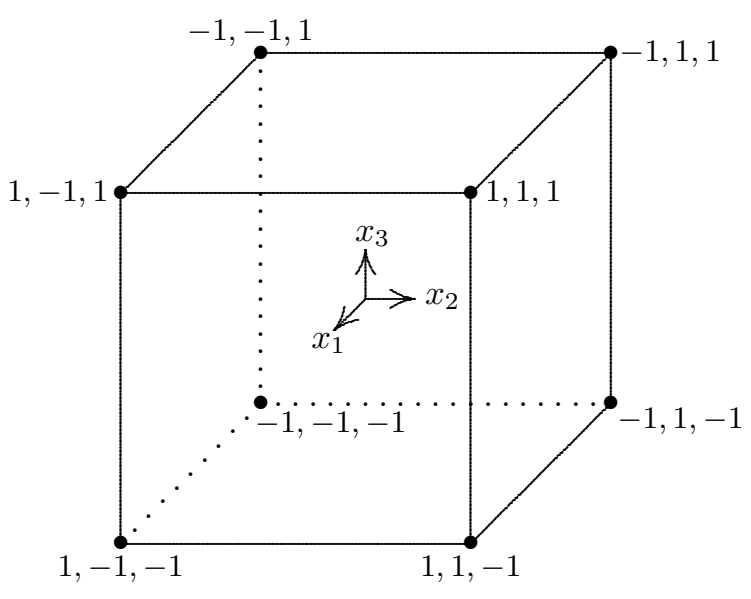

Figure 3.

Using the fact that the tensor product trapezoidal rule will exactly integrate a multilinear $u_{h}$ over the faces of $\Gamma(\mathcal{E})$, we have

$$
\begin{aligned}
\left(\left(u_{h}\right)_{\alpha}, 1\right)_{\mathcal{E}} & =\sum_{i=1}^{N} \alpha_{i}\left(\int_{\Gamma_{i}^{+}} u_{h}-\int_{\Gamma_{i}^{-}} u_{h}\right) \\
& =\left(\frac{h}{2}\right)^{N-1} \sum_{i=1}^{N} \alpha_{i} \sum_{\hat{\mathbf{x}} \in V} \hat{x}_{i} \hat{u}_{h}(\hat{\mathbf{x}})
\end{aligned}
$$

where $\Gamma_{i}^{+}\left(\Gamma_{i}^{-}\right)$denotes the face of $\Gamma(\mathcal{E})$ for which $\hat{x}_{i}=+1(-1)$. Hence in the case $k=1$, we get

$$
\left(\frac{h}{2}\right)^{N-1} \sum_{\hat{\mathbf{x}} \in V}\left(\sum_{i=1}^{N} \alpha_{i} \hat{x}_{i}\right) \hat{u}_{h}(\hat{\mathbf{x}})=(f, 1)_{\mathcal{E}}
$$

This gives

$$
\hat{u}_{h}(1,1, \ldots, 1)=\frac{-\sum_{\hat{\mathbf{x}} \in V, \hat{\mathbf{x}} \neq(1,1, \ldots, 1)}\left(\sum_{i=1}^{N} \alpha_{i} \hat{x}_{i}\right) \hat{u}_{h}(\hat{\mathbf{x}})+\left(\frac{2}{h}\right)^{N-1}(f, 1)_{\mathcal{E}}}{\sum_{i=1}^{N} \alpha_{i}} .
$$

We now show by means of a counterexample that the stability bound (1.5) does not hold for linear approximation for $N>2$. We partition the vertex set $V$ into $\left\{V_{k}\right\}_{k=0}^{N}$, where $V_{k}$ consists of those vertices $\left(\hat{x}_{1}, \ldots, \hat{x}_{N}\right) \in V$ for which exactly $k$ $\hat{x}_{j}$ 's have value +1 . The scheme can then be written

$$
\sum_{i=1}^{N} \alpha_{i} \sum_{k=0}^{N} \sum_{\hat{\mathbf{x}} \in V_{k}} \hat{x}_{i} \hat{u}_{h}(\hat{\mathbf{x}})=2^{N-1} h \bar{f}
$$

where $\bar{f}$ is the average value of $f(\mathbf{x})$ over $\mathcal{E}$. If $\hat{u}_{h}(\hat{\mathbf{x}})$ had the same value for all $\hat{x} \in V_{k}, \hat{u}_{h}^{k}$, say, then (3.3) could be written

$$
\left(\sum_{i=1}^{N} \alpha_{i}\right)\left(\sum_{k=0}^{N} \hat{u}_{h}^{k} \sum_{\hat{\mathbf{x}} \in V_{k}} \hat{x}_{i}\right)=2^{N-1} h \bar{f}
$$


where we have used the fact that $\sum_{\hat{\mathbf{x}} \in V_{k}} \hat{x}_{i}$ is independent of $i$ via symmetry. Note that $V_{k}$ consists of $\left(\begin{array}{c}N \\ k\end{array}\right)$ vertices, and for $\hat{\mathbf{x}} \in V_{k}, \sum_{i=1}^{N} \hat{x}_{i}=2 k-N$. Therefore,

$$
\sum_{\hat{\mathbf{x}} \in V_{k}} \hat{x}_{i}=\frac{1}{N} \sum_{i=1}^{N} \sum_{\hat{\mathbf{x}} \in V_{k}} \hat{x}_{i}=\frac{1}{N} \sum_{\hat{\mathbf{x}} \in V_{k}} \sum_{i=1}^{N} \hat{x}_{i}=\frac{1}{N}(2 k-N)\left(\begin{array}{l}
N \\
k
\end{array}\right) .
$$

Thus (3.4) can be reduced to

$$
\bar{\alpha} \sum_{k=0}^{N}(2 k-N)\left(\begin{array}{l}
N \\
k
\end{array}\right) \hat{u}_{h}^{k}=2^{N-1} h \bar{f}, \quad \bar{\alpha} \equiv \frac{1}{N} \sum_{i=1}^{N} \alpha_{i} .
$$

Under the assumption that $\bar{f}$ has a common value, $\bar{f}_{j}$, for all $\mathcal{E} \subset S_{j}, j=0,1, \ldots$, we look for solutions over the unit cube $\Omega$ that are the same throughout each layer, i.e.,

$$
u_{h}\left(i_{1} h, \ldots, i_{N} h\right)=U\left(i_{1}+\cdots+i_{N}\right)
$$

in conformity with (3.5). Then, for an element $\mathcal{E} \subset S_{j}$, we have $\hat{u}_{h}^{k}=U(j+k)$ and (3.5) becomes

$$
\sum_{k=0}^{N}(2 k-N)\left(\begin{array}{l}
N \\
k
\end{array}\right) U(j+k)=\frac{2^{N-1} h}{\bar{\alpha}} \bar{f}_{j}
$$

As special cases, we have

$$
\begin{aligned}
2\left(U_{j+2}-U_{j}\right) & =(2 h / \bar{\alpha}) \bar{f}_{j}, & & \text { for } N=2, \\
3\left(U_{j+3}+U_{j+2}-U_{j+1}-U_{j}\right) & =(4 h / \bar{\alpha}) \bar{f}_{j}, & & \text { for } N=3, \\
4\left(U_{j+4}+2 U_{j+3}-2 U_{j+1}-U_{j}\right) & =(8 h / \bar{\alpha}) \bar{f}_{j}, & & \text { for } N=4 .
\end{aligned}
$$

In general, the characteristic polynomial associated with the difference equation (3.6) is $\phi(x)=\sum_{k=0}^{N}(2 k-N)\left(\begin{array}{c}N \\ k\end{array}\right) x^{k}$. We will show that it can also be written

$$
\phi(x)=N(x-1)(x+1)^{N-1} .
$$

Note first that

$$
\begin{aligned}
\left(\begin{array}{c}
N-1 \\
k-1
\end{array}\right)-\left(\begin{array}{c}
N-1 \\
k
\end{array}\right) & =\frac{(N-1) !}{(k-1) !(N-k) !}-\frac{(N-1) !}{k !(N-k-1) !} \\
& =\frac{(N-1) !}{k !(N-k) !}(k-(N-k)) \\
& =\frac{2 k-N}{N}\left(\begin{array}{c}
N \\
k
\end{array}\right) .
\end{aligned}
$$


Thus

$$
\begin{aligned}
(x-1)(x+1)^{N-1} & =(x-1) \sum_{k=0}^{N-1}\left(\begin{array}{c}
N-1 \\
k
\end{array}\right) x^{k} \\
& =\sum_{k=1}^{N}\left(\begin{array}{c}
N-1 \\
k-1
\end{array}\right) x^{k}-\sum_{k=0}^{N-1}\left(\begin{array}{c}
N-1 \\
k
\end{array}\right) x^{k} \\
& =x^{N}-1+\sum_{k=1}^{N-1}\left[\left(\begin{array}{c}
N-1 \\
k-1
\end{array}\right)-\left(\begin{array}{c}
N-1 \\
k
\end{array}\right)\right] x^{k} \\
& =\sum_{k=0}^{N}\left(\frac{2 k-N}{N}\right)\left(\begin{array}{c}
N \\
k
\end{array}\right) x^{k} .
\end{aligned}
$$

Having established the equivalence of the two forms for $\phi(x)$, we immediately conclude that (3.6) with $\bar{f}_{j} \equiv 0$ has solutions

$$
U_{j}=1, \quad U_{j}=(-1)^{j} j^{m}, m=0, \ldots, N-2 .
$$

Of these $N$ solutions, all but the first are spurious. Note, in particular, that for $N \geq 3$ the mode $U_{j}=(-1)^{j} j^{N-2}$ is unstable.

It is instructive to look at the finite element solution corresponding to the most rapidly growing mode in the case $N=3$ (i.e., $U_{j}=(-1)^{j} j$ ). It is, in $\mathcal{E}_{i_{1}, i_{2}, i_{3}}$,

$$
\begin{aligned}
\hat{u}_{h}\left(\hat{x}_{1}, \hat{x}_{2}, \hat{x}_{3}\right)=(-1)^{j}\left[\left(j+\frac{3}{2}\right) \hat{x}_{1} \hat{x}_{2} \hat{x}_{3}-\frac{1}{2}\left(\hat{x}_{1} \hat{x}_{2}+\hat{x}_{2} \hat{x}_{3}+\hat{x}_{3} \hat{x}_{1}\right)\right], & \\
j & =i_{1}+i_{2}+i_{3} .
\end{aligned}
$$

Each of the functions comprising $\hat{u}_{h}-\hat{x}_{1} \hat{x}_{2} \hat{x}_{3}, \hat{x}_{1} \hat{x}_{2}, \hat{x}_{2} \hat{x}_{3}$, and $\hat{x}_{3} \hat{x}_{1}$-has $\alpha$ derivative which is orthogonal to constants over $\mathcal{E}_{i_{1}, i_{2}, i_{3}}$. Thus these functions are not controlled directly by the inner product conditions (1.4), and this is the source of the instability.

We conclude by giving a specific counterexample to (1.5) for the case $N=3$. Assuming the unit cube is divided into $n^{3}$ cubical elements, the corresponding layers and vertex values of $u_{h}$ are $S_{0}, \ldots, S_{3 n-3}$ and $U_{0}, \ldots, U_{3 n}$. Let $u_{h}=0$ on $\Gamma_{\text {in }}(\Omega)$ and

$$
f(\mathbf{x})=\left\{\begin{array}{c}
-1, \mathbf{x} \in S_{2 n-2}, \\
+1, \mathbf{x} \in S_{2 n-1} \\
0, \text { otherwise }
\end{array}\right.
$$

The solution is then

$$
U_{j}=\left\{\begin{array}{l}
0, j=0,1, \ldots, 2 n, \\
(4 h / 3 \bar{\alpha})(-1)^{j-2 n}(j-2 n), j=2 n+1, \ldots, 3 n,
\end{array}\right.
$$

which gives $\left|u_{h}\right|_{\Gamma_{\text {out }}(\Omega)}=O(1)$ for a forcing function of size $\|f\|_{\Omega}=O(\sqrt{h})$.

\section{ACKNOWLEDGMent}

I thank Endre Süli for comments on an earlier version of this manuscript. 


\section{REFERENCES}

1. P. Balland and E. Süli, Analysis of the cell-vertex finite volume method for hyperbolic problems with variable coefficients, SIAM J. Numer. Anal. 34 (1997), 1127-1151. MR 98i:65089

2. P. I. Crumpton and G. J. Shaw, Cell vertex finite volume discretizations in three dimensions, Internat. J. Numer. Meth. Fluids 14 (1992), 505-527. MR 92k:76060

3. R. S. Falk and G. R. Richter, Analysis of a continuous finite element method for hyperbolic equations, SIAM J. Numer. Anal. 24 (1987), 257-278. MR 88d:65133

4. R. S. Falk and G. R. Richter, Local error estimates for a finite element method for hyperbolic and convection-diffusion equations, SIAM J. Numer. Anal. 29 (1992), 730-754. MR 93e:65137

5. R. S. Falk and G. R. Richter, Explicit finite element methods for symmetric hyperbolic systems, SIAM J. Numer. Anal. 36 (1999), 935-952. MR 2000b:65181

6. C. Johnson and J. Pitkäranta, An analysis of the discontinuous Galerkin method for a scalar hyperbolic equation, Math. Comp. 46 (1986), 1-26. MR 88b:65109

7. P. Lesaint and P. A. Raviart, On a finite element method for solving the neutron transport equation, Mathematical Aspects of Finite Elements in Partial Differential Equations, (C. deBoor, ed.), Academic Press, 1974, pp. 89-123. MR 58:31918

8. K. W. Morton, M. Stynes, and E. Süli, Analysis of a cell-vertex finite volume method for convection-diffusion problems, Math. Comp. 66 (1997), 1389-1406. MR 98a:65103

9. W. H. Reed and T. R. Hill, Triangular mesh methods for solving the neutron transport equation, Los Alamos Scientific Laboratory Report LA-UR-73-479..

10. B. Wendroff, On centered difference approximations for hyperbolic systems, J. Soc. Indust. Appl. Math. 8 (1960), 549-555. MR 22:7259

11. R. Winther, A stable finite element method for initial-boundary value problems for first-order hyperbolic systems, Math. Comp. 36 (1981), 65-86. MR 81m:65181

Department of Computer Science, Rutgers University, New Brunswick, New Jersey 08903

E-mail address: richter@cs.rutgers.edu 